\title{
Use of the Pastorex aspergillus antigen latex agglutination test for the diagnosis of invasive aspergillosis
}

\author{
V Hopwood, E M Johnson, J M Cornish, A B M Foot, E G V Evans, D W Warnock
}

PHLS Mycology

Reference Laboratory, Department of Microbiology,

General Infirmary,

General Infirmary,

Leeds LS2 9JT

$\checkmark$ Hopwood

E G V Evans

PHLS Mycology Reference Laboratory, Bristol Public Health Laboratory, Bristol BS2 8EL

E M Johnson

D W Warnock

Bone Marrow

Transplant Unit,

Royal Hospital

for Sick Children,

Bristol BS2 8BU

J M Cornish

A B M Foot

Correspondence to: Correspondence
Dr V Hopwood.

Accepted for publication 4 August 1994

\begin{abstract}
Aims-To evaluate the Pastorex aspergillus antigen latex agglutination test for the diagnosis of invasive aspergillosis in patients undergoing liver or bone marrow transplantation.

Methods-Serum samples were taken at least twice weekly post-transplant and tested for Aspergillus antigen. Latex agglutination test results were compared with microbiological examination of respiratory, urine and bile specimens. Serum samples from liver transplant patients were also tested for antibodies to Aspergillus fumigatus by counter immunoelectrophoresis.
\end{abstract}

Results-Eight of the 91 patients studied developed invasive aspergillosis. Positive latex agglutination tests were obtained in eight of $187(4.3 \%)$ serum samples from four of these eight patients. The other four patients with invasive aspergillosis gave consistently negative latex agglutination tests. A positive latex agglutination test was the first indication of invasive aspergillosis in two patients; these patients were already on amphotericin B. Positive latex agglutination tests were the only evidence of invasive aspergillosis in one patient who subsequently died of the infection. False positive latex agglutination tests were obtained in five of $83(6 \%)$ patients with no evidence of invasive aspergillosis and misleading positive cultures seen in nine of 83 $(10 \cdot 8 \%)$. No antibodies were detected in three of four liver transplant patients with invasive aspergillosis. Conversely, antibodies were detected in 63 of 262 (24\%) serum samples from 43 liver transplant patients with no evidence of invasive aspergillosis; one of these patients had an antibody titre of $1: 2$ on four separate occasions.

Conclusions-The Pastorex aspergillus antigen latex agglutination test, when used alone, lacks sensitivity and specificity for the early diagnosis of invasive aspergillosis. A diagnosis was made in all patients with invasive aspergillosis when both culture and antigen tests were performed although using these criteria a false positive diagnosis would have been made in 13 of $83(15.6 \%)$ patients. Microbiological and serial serological investigations for antigen should both be performed and the results considered in conjunction with radiological and clinical evidence.

(f Clin Pathol 1995;48:210-213)

Keywords: Aspergillus, antigen test, invasive aspergillosis.

Invasive aspergillosis is a major cause of morbidity and mortality in immunocompromised patients undergoing intensive chemotherapy for cancer or organ transplantation. The response of immunosuppressed patients with invasive aspergillosis to specific antifungal therapy is poor, with an overall mortality rate of $45 \%$ and a mortality rate in bone marrow transplant recipients of $94 \% .{ }^{1}$ The major problem associated with invasive aspergillosis is the difficulty in diagnosing this infection early enough to be of value in patient management. Cultures of blood or respiratory specimens are seldom positive especially during the early stages of the disease. Aisner $e t a l^{2}$ demonstrated mortality of $83 \%$ in six patients where there was early evidence of pulmonary aspergillosis whereas mortality was $100 \%$ in patients whose infection was diagnosed late.

At present, reliable antemortem diagnosis of invasive aspergillosis requires invasive procedures to obtain biopsy material for histological and microbiological examination. However, clinicians are often reluctant to perform these procedures because of the associated morbidity and mortality.

Serological procedures are used as an adjunct to microbiological methods for the diagnosis of invasive aspergillosis but current tests lack sensitivity. Antibody detection is often negative because of the fulminant nature of the disease or the poor immunological status of the host, or both. ${ }^{3}$ Antigen detection tests have been developed ${ }^{34}$ but most have only been evaluated in individual laboratories. Recently, however, a monoclonal antibody based latex particle agglutination test for the detection of Aspergillus galactomannan in serum has become available commercially. We report our experience with this test, over two years, for the routine surveillance of patients undergoing liver and bone marrow transplantation.

\section{Methods}

MYCOLOGICAL SURVEILLANCE

Forty seven liver transplant recipients were screened for fungal infection over two years. 
Table 1 Antigen test results in patients with invasive aspergillosis

\begin{tabular}{|c|c|c|c|c|}
\hline \multirow[b]{2}{*}{ Case No. } & \multicolumn{2}{|c|}{ No. of blood samples } & \multirow[b]{2}{*}{ Other positive samples } & \multirow[b]{2}{*}{ Outcome } \\
\hline & Tested & Positive & & \\
\hline $\begin{array}{l}1 \text { (LT) } \\
2 \text { (LT) } \\
3 \text { (LT) } \\
4 \text { (LT) } \\
5 \text { (BMT) } \\
6 \text { (BMT) } \\
7 \text { (BMT) } \\
8 \text { (BMT) }\end{array}$ & $\begin{array}{r}17 \\
2 \\
4 \\
8 \\
45 \\
58 \\
31 \\
22\end{array}$ & $\begin{array}{l}0 \\
2 \text { (weeks } 4 \text { and 5) } \\
0 \\
2 \text { (week 6) } \\
2 \text { (weeks } 4 \text { and 20) } \\
2 \text { (week 22) } \\
0 \\
0\end{array}$ & $\begin{array}{l}\text { BAL + TA (weeks } 25 \text { and 26) } \\
\text { Sputum (week 4) } \\
\text { Bile, urine (weeks } 4 \text { and 5) } \\
\text { Sputum (week 6) } \\
\text { Nil } \\
\text { BAL (week 9) } \\
\text { Nasal swab (week 6) } \\
\text { Pus (week 9) }\end{array}$ & $\begin{array}{l}\text { Died (week 26) } \\
\text { Died (week 5) } \\
\text { Died (week 6) } \\
\text { Died (week 6) } \\
\text { Died (week 22) } \\
\text { Died (week 22) } \\
\text { Died (week 24) } \\
\text { Died (week 11) }\end{array}$ \\
\hline
\end{tabular}

a Invasive aspergillosis confirmed at necropsy.

${ }^{b}$ Invasive aspergillosis strongly suspected from radiological and mycological evidence but permission for necropsy refused. LT, liver transplant; BMT, bone marrow transplant; TA, tracheal aspirate.

Clinical specimens (bile, urine, serum) were taken for mycological investigation twice weekly post-transplant until discharge or death. Other specimens (sputum, bronchoalveolar lavage (BAL) fluid, tracheal aspirates) were obtained if there was clinical suspicion of invasive aspergillosis. All specimens were centrifuged and the deposit examined for the presence of fungi by direct microscopy in $\mathrm{KOH}$ and plated on to Sabouraud agar containing $0.5 \%$ chloramphenicol. Plates were examined for fungal growth after incubation at both $27^{\circ} \mathrm{C}$ and $37^{\circ} \mathrm{C}$ for 48 hours (urine and bile) or 96 hours (respiratory specimens).

Serum samples were tested weekly for antibodies to $A$ fumigatus by counterimmunoelectrophoresis ${ }^{5}$ against $A$ fumigatus somatic and culture filtrate antigens (Mercia Diagnostics Ltd., Guildford, UK) at concentrations of $20 \mathrm{mg} / \mathrm{ml}$.

Serum samples were also tested for $A$ spergillus antigen if (i) the patient was pyrexial, (ii) the patient had any signs of chest infection, (iii) an Aspergillus species had been isolated from any clinical specimen during routine mycological screening, or (iv) the patient was on the intensive care unit.

Forty four patients undergoing allogeneic bone marrow transplantation were screened for fungal infection over two years. Serum specimens were collected twice weekly post-transplant until discharge or death. Other specimens (nasal swabs, sputum, BAL fluid) were taken at intervals. If invasive aspergillosis was suspected on clinical or mycological grounds, daily testing of serum for Aspergillus antigen and empirical treatment with amphotericin B were instituted. Antibody testing was not performed as it was considered that this would not yield useful information.

\section{ASPERGILLUS ANTIGEN TEST}

The aspergillus latex agglutination test ( $\mathrm{Pa}-$ storex aspergillus; Sanofi, Diagnostics Pasteur, Paris, France) was carried out according to the manufacturer's instructions with slight modification using half volumes of reagents. Preliminary studies using serial dilutions of positive control antigen and patients' serum samples showed that these modifications did not affect the sensitivity of the test. Briefly, $300 \mu \mathrm{l}$ of serum was mixed with $100 \mu l$ of treatment solution and boiled for three minutes. After centrifugation at $15000 \mathrm{rpm}$ for 10 minutes the supernatant was tested for galactomannan by placing $20 \mu$ of specimen and $5 \mu$ of sensitised latex reagent on a clean black slide (manufacturer's instructions: $40 \mu \mathrm{l}$ specimen and $10 \mu$ l latex). After mixing thoroughly with a plastic mixing stick the slide was placed on a rocking table and agglutination recorded after five minutes. Because of previous reports of false positive agglutination tests with these reagents, ${ }^{67}$ all specimens giving positive reactions were retested and were only regarded as true positives if the results were reproducible. Agglutination was scored on a scale of $1+$ to $3+$.

\section{Results}

Over the two years, 279 serum samples from liver transplant recipients were tested for Aspergillus antigen; five $(1 \cdot 8 \%)$ positive tests were observed.

Two patients (cases 3 and 4) developed invasive aspergillosis, confirmed at necropsy, and two (cases 1 and 2) had presumed invasive aspergillosis although a request for necropsy was refused. Aspergillus antigen tests were performed on 31 serum samples from these four patients with four (13\%) positive results (table 1). Twenty one serum samples from cases 1 and 3 all gave negative antigen test results. $A$ fumigatus was isolated from BAL fluid and two tracheal aspirate samples from case 1 , who died 12 days after the first positive culture. Invasive aspergillosis was confirmed at necropsy in case 3 with widespread involvement of the heart valves, myocardium, kidney, and thyroid.

Two serum samples from each of the other two cases were antigen positive (table 1). Culture of $A$ fumigatus preceded the first positive antigen test by two days in case 2 but occurred four days after the first positive antigen test in case 4 . Both patients died within four days of the first positive antigen tests. A necropsy performed on case 4 revealed extensive invasive aspergillosis with involvement of the lungs, brain, kidneys, and thyroid.

In all four patients empirical antifungal therapy with amphotericin B had been instituted several days before confirmation of Aspergillus infection by either culture or antigen testing.

Of the 43 liver transplant recipients without invasive aspergillosis, one false positive antigen test ( $1+$ agglutination) was observed out of 248 serum samples tested $(0 \cdot 4 \%)$. This occurred in a 13 year old boy (case 9; table 2) who had undergone liver transplantation as a sequel to cystic fibrosis; he was known to be colonised with both $A$ fumigatus and $A$ terreus. Of the 
Table 2 Antigen test results in other patients

\begin{tabular}{|c|c|c|c|c|}
\hline \multirow[b]{2}{*}{ Case No. } & \multicolumn{2}{|c|}{ No. of blood samples } & \multirow[b]{2}{*}{ Other samples positive } & \multirow[b]{2}{*}{ Outcome } \\
\hline & Tested & Positive & & \\
\hline 9 (LT) & 31 & 1 & Sputum $^{\mathrm{f}}$ & Now $29 / 12$ post-LT \\
\hline $10(\mathrm{LT})$ & 4 & 0 & Bile (week 2) & Now $24 / 12$ post-LT \\
\hline 11 (LT) & 9 & 0 & Urine (weeks 7 and 8) & Now $11 / 12$ post-LT \\
\hline $12(\mathrm{LT})$ & 20 & 0 & Sputum (week 1) & Died (week 42) \\
\hline $13(\mathrm{LT})$ & 7 & 0 & Sputum (weeks 3-5) & Now $12 / 12$ post-LT \\
\hline $14(\mathrm{LT})$ & 4 & 0 & Bile, urine (weeks 2 and 3 ) & Now $8 / 12$ post-LT \\
\hline $15(B M T)^{a}$ & 13 & 1 (week 5) & Nil & Now $27 / 12$ post-BMT ${ }^{b}$ \\
\hline $16(\mathrm{BMT})^{\mathrm{a}}$ & 46 & 1 (week 4) & Nil & Died (week 29) \\
\hline $17(\mathrm{BMT})^{\mathrm{a}}$ & 9 & 1 (week 3) & Nil & Now $11 / 12$ post-BMT ${ }^{b}$ \\
\hline $18(\mathrm{BMT})^{\mathrm{a}}$ & 17 & 2 (weeks 4 and 6) & Nil & Now $4 / 12$ post-BMT \\
\hline 19 (BMT) & 26 & 0 & BAL (week 15) & Died (week 35) ${ }^{\mathrm{c}, \mathrm{e}}$ \\
\hline $20(\mathrm{BMT})$ & 25 & 0 & Sputum (week 28) & Now $23 / 12$ post-BMT \\
\hline 21 (BMT) & 21 & 0 & BAL (week 7) & Now $18 / 12$ post-BMT ${ }^{b}$ \\
\hline
\end{tabular}

a No clinical, mycological or radiological evidence of invasive aspergillosis.

b No antifungal therapy given.

${ }^{c}$ No evidence of invasive aspergillosis at necropsy.

d Amphotericin B given on the basis of antigen result.

' Patient treated with itraconazole on basis of culture result.

f Patient with cystic fibrosis. Colonised with $A$ fumigatus and $A$ terreus.

antigen tests performed on 31 serum samples from this patient, only one was positive.

$A$ fumigatus was isolated from five other liver transplant recipients (cases 10 to 14 ; table 2), four of whom were clinically well two to six months after transplantation without administration of antifungal drugs. One patient (case 12) died 42 weeks after transplantation with no clinical or radiological evidence of invasive aspergillosis.

Of 467 serum samples tested for antibodies to $A$ fumigatus, 65 (14\%) were positive. Only one of the four patients with invasive aspergillosis (table 1; case 3 ) had weakly detectable antibodies in two of five serum samples tested; the appearance of antibodies preceded the first positive culture of $A$ fumigatus by 14 days. No antibodies were detected in any of the other three liver transplant patients with invasive aspergillosis.

Three of the six patients with no clinical evidence of invasive aspergillosis, but from whom $A$ fumigatus was isolated, became weakly antibody positive at some stage (15 of 66 serum samples; cases 9, 10 and 12); the remainder had no detectable antibodies.

Seventeen other patients had positive antibody tests (48 of 196 serum samples, 24\%), and one patient had an antibody titre of $1: 2$ on four occasions despite having no clinical or mycological evidence of invasive aspergillosis.

Serum samples $(n=680)$ from patients undergoing bone marrow transplantation were tested with the latex agglutination reagent. Four patients had invasive aspergillosis confirmed at necropsy (cases 5 to 8; table 1). Four positive aspergillus antigen tests were obtained in 156 serum samples from these patients $(2.6 \%$; two were unconfirmed as there was insufficient specimen for repeat testing). All four positive tests were on serum samples from cases 5 and 6 , and the aspergillus antigen test was the first indication of fungal infection in case 5, although positive agglutination was not confirmed. A fumigatus was isolated from BAL fluid from case 6 at nine weeks post-transplant, at which time antifungal chemotherapy with amphotericin B was instituted. Positive aspergillus antigen tests were not obtained on this patient until 22 weeks post-transplant, the last week of life.
Cases 7 and 8 did not give a positive antigen test despite 53 serum samples being screened. Case 7 had $A$ fumigatus isolated from a nasal swab at six weeks post-transplant and died 24 weeks post-transplant. Case 8 developed a cerebral abscess from which $A$ fumigatus was isolated at week 9 and died two weeks later.

Positive aspergillus antigen tests were also obtained on four other patients (cases 15 to 18), although they had no mycological, radiological or clinical evidence of invasive aspergillosis (table 2). Two of these patients remain well, 27 and 11 months after transplantation, despite receiving no antifungal therapy. One patient died but had no evidence of invasive aspergillosis at necropsy and one patient received amphotericin B on the basis of the antigen result and was well at the time of writing.

Thirty six patients (439 serum samples) had negative antigen test results. $A$ fumigatus was recovered from specimens from three of these patients (table 2). Case 19 was treated with itraconazole following isolation of $A$ fumigatus from BAL fluid 15 weeks post-transplant. Although the patient died 35 weeks posttransplant there was no evidence of invasive aspergillosis at necropsy. The other two patients (cases 20 and 21) remain well 23 and 18 months post-transplant, respectively, although case 20 did receive a four week course of amphotericin $B$.

\section{Discussion}

Reliable diagnosis of invasive aspergillosis is difficult and is often only made at necropsy. Aspergillus is only recovered from sputum in $8-34 \%$ of patients and sputum samples are often scanty or unavailable. ${ }^{8}$ In some cases recovery of Aspergillus from sputum may be because of contamination rather than infection; culture of BAL fluid or tracheal aspirate specimens may give more accurate results. In our series of patients $A$ fumigatus was isolated from respiratory specimens (nasal swab, sputum, BAL fluid, tracheal aspirate) from five of the eight $(62 \%)$ patients with invasive aspergillosis but also from six of $83(7 \cdot 2 \%)$ patients with no evidence of invasive aspergillosis.

Antibody detection lacked both sensitivity and specificity. Only one of our liver transplant 
patients with confirmed invasive aspergillosis had detectable antibodies to $A$ fumigatus whereas antibody tests were positive in 20 of 42 patients with no evidence of invasive aspergillosis; one of these latter patients had an antibody titre of $1: 2$ on at least four occasions. It is unlikely that the presence of antibodies to Aspergillus affected antigen detection as heat treatment of serum before testing with the latex agglutination reagent would dissociate any immune complexes.

In this prospective study the latex agglutination aspergillus antigen detection test lacked sufficient sensitivity for the early diagnosis of invasive aspergillosis, which would influence treatment, but was of value in helping to confirm a diagnosis. The antigen test was positive in four of the eight $(50 \%)$ patients with invasive aspergillosis and was the first indicator of infection in two patients; all of the patients with invasive aspergillosis in this study, however, had been receiving empirical antifungal chemotherapy with amphotericin B for several days before confirmation of infection by either culture or antigen testing. Two positive latex agglutination tests were the only evidence of invasive aspergillosis in a bone marrow transplant recipient who subsequently died of the infection.

Confirmed false positive latex agglutination tests were obtained in five of 83 (6\%) cases. The reasons for these false positive antigen tests are not known although it has been suggested that contamination of serum samples with some airborne moulds, ${ }^{9}$ or bacteria, ${ }^{10}$ may result in cross-reactivity with the monoclonal antibody used in the kit. Care was taken with other factors which may interfere with the test ${ }^{11}$; all serum samples were separated from the clot well within 24 hours of sampling, microcentrifuge tubes were properly sealed during boiling and, as the volumes of reagents were halved, the serum-latex mixtures were covered during mixing to prevent dessication.

Antigen detection tests for the diagnosis of invasive aspergillosis can give more meaningful results by testing sequential rather than single serum samples. ${ }^{4}$ However, in this study, four patients with invasive aspergillosis gave consistently negative antigen test results despite testing multiple samples.

The diagnosis of invasive aspergillosis was enhanced when antigen tests and culture were both performed; a positive culture and/or antigen test occurred in all eight patients with invasive aspergillosis. However, using these criteria a false positive diagnosis would also have been made in $16 \%(13 / 83)$ of patients with no clinical or radiological evidence of invasive aspergillosis.
Clearly, methods for the diagnosis of invasive aspergillosis still need to be improved. Urine ${ }^{12}$ and BAL fluid ${ }^{13}$ have both been shown to contain detectable antigen in patients with invasive aspergillosis and these may be better specimens to test than serum. Unfortunately, the latex agglutination reagent evaluated in this study is only suitable for use on serum samples. Until more reliable tests such as the polymerase chain reaction are developed for the diagnosis of invasive aspergillosis, the Pastorex aspergillus antigen latex agglutination test alone should not be relied on to give an early diagnosis of invasive aspergillosis and users of the test should recognise its limitations and interpret its results with care.

Despite the disappointing performance of this latex agglutination test, we still believe that both microbiological and serial serological investigations for aspergillus antigen should be performed to maximise the chances of diagnosing invasive aspergillosis and the results considered in conjunction with radiological and clinical evidence of infection.

The authors would like to acknowledge the help of the staff of the Liver Transplant Unit, St James's University Hospital, Leeds, and the Bone Marrow Transplant Unit, Bristol Royal Hospital for Sick Children, for collecting specimens and their help in providing clinical information, and Dr J I Wyatt, Consultant Pathologist, St James's University Hospital, Leeds, for providing the necropsy data.

1 Denning DW, Stevens, DA. Antifungal and surgical treatment of invasive aspergillosis: review of 2121 published cases. Rev Infect Dis 1990;12:1147-201.

2 Aisner J, Wiernik PH, Schimpff SC. Treatment of invasive aspergillosis: relation of early diagnosis and treatment to response. Ann Intern Med 1977;86:539-43.

3 Hopwood V, Evans EGV. Serological tests in the diagnosis and prognosis of fungal infection in the compromised patient. In: Warnock DW, Richardson MD, eds. Fungal infection in the compromised patient. 2nd edn. Chichester: John Wiley \& Sons, 1991:311-53.

4 Wilson EV, Hearn VM, Mackenzie DWR. Evaluation of a test to detect circulating Aspergillus fumigatus antigen in a survey of immunocompromised patients with proven or a survey of immunocompromised patients with proven or suspected

5 Evans EGV, ed. Serology of Fungal Infection and Farmer's Lung Disease. A Laboratory Manual. University Printing Service, University of Leeds, Leeds.

6 Warnock DW, Foot ABM, Johnson EM, Mitchell SB, Cornish JM, Oakhill A. Aspergillus antigen latex test for diagnosis of invasive aspergillosis. Lancet 1991;338:1023-4.

7 Knight F, Mackenzie DWR. Aspergillus antigen latex test for diagnosis of invasive aspergillosis. Lancet 1992;339: 188.

8 Cairns MR, Durack DT. Fungal pneumonia in the immunocompromised host. Semin Respir Med 1986;1:16685

9 Kappe R, Schulze-Berge A. New cause for false-positive results with the Pastorex Aspergillus antigen latex agresults with the Pastorex Aspergillus antigen late

10 Dupont B, Improvisi L, Povost F. Detection de galactomannane dans les aspergilloses invasives humaines et animales avec un test au latex. Bull Soc Fr Mycol Med 1990;19:35-42.

11 Stynen D, Meulemans L, Garrigues ML. Aspergillus antigen latex test for diagnosis of invasive aspergillosis. Lancet 1992;339:188.

12 Haynes KA, Latgé J-P, Rogers TR. Detection of Aspergillus antigens associated with invasive infection. $\mathcal{F}$ Clin Microbiol 1990;28:2040-4.

13 Andrews CP, Weiner MH. Aspergillus antigen detection in bronchoalveolar lavage fluid from patients with invasive aspergillosis and aspergillomas. Am f Med 1982;73:37280 . 